\title{
reviscafuences
}

ISSN: 1575-7072 | e-ISSN: 2172-7775

Páginas: 1-14

Recibido: 2019-03-18

Revisado: 2020-07-08

Aceptado: 2020-07-27

Preprint: 2020-07-31

Publicación Final: 2020-09-15

DOI:https://doi.org/10.12795/revistafuentes.2020.v22.i2.08

\section{Relación entre aspectos neuropsicológicos cognitivos y socioemocionales en alumnos de Educación Primaria}

\section{Relationship between neuropsychological cognitive and socioemotional aspects in Primary School students}

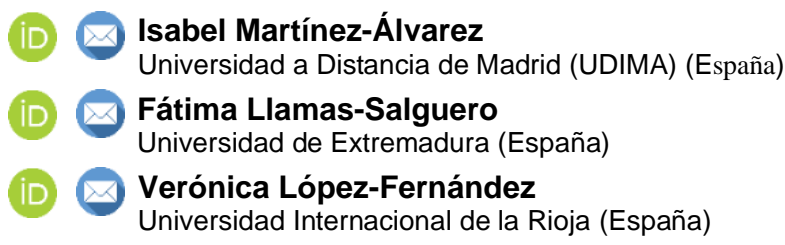

\section{Resumen}

Este estudio analiza la relación entre diferentes aspectos neuropsicológicos y educativos como son el rendimiento académico las competencias, la comprensión lectora, la empatía, la creatividad y el autoconcepto. El objetivo general es estudiar la relación entre las variables neurocognitivas y socioemocionales implicadas en el proceso de enseñanza-aprendizaje. Participaron 82 alumnos/as, 42 niños y 40 niñas, de tercero de Educación Primaria de un colegio concertado de Ávila (España). Como instrumentos y materiales se analizan el rendimiento académico, la prueba de evaluación individual, ACL (evaluación de la comprensión lectora), BES-A (Escala de empatía básica abreviada), CREA (Inteligencia Creativa) y un cuestionario de autoconcepto. Se realizó un análisis descriptivo de las variables de estudio recurriendo al cálculo de la media y la desviación típica. Posteriormente, se calcularon las correlaciones entre variables a través del índice de correlación de Pearson. Finalmente, para la comparación entre grupos se realizaron ANOVA de un factor. Entre los resultados se encontraron relaciones entre el rendimiento, las competencias y la comprensión lectora, entre la calificación de la asignatura de matemáticas y el nivel de empatía cognitiva, y el autoconcepto, y entre la creatividad y la competencia matemática y la comprensión lectora crítica. Al comparar alumnos con rendimiento medio y alto se encontraron diferencias en su nivel de comprensión lectora y autoconcepto. Los alumnos con baja y alta creatividad difieren en su nivel de competencia matemática. Queda latente la necesidad de diseñar programas de intervención neuropsicológicos que trabajen de manera conjunta todas estas variables, en aras de lograr un desarrollo integral de nuestros alumnos, tanto en aspectos cognitivos, como emocionales y sociales.

Abstract

This study analyzes the relationship between different neuropsychological and educational aspects such as academic performance, competences, reading comprehension, empathy, creativity and self-concept. The general objective is to study the relationship between the neurocognitive and socio-emotional variables involved in the teaching-learning process. 82 students participated, 42 men and 40 women, from the 3rd year of Primary Education of a concerted school in Avila (Spain). As instruments and materials, academic performance, the individual assessment test, ACL (reading comprehension evaluation), BES-A (Short Basic Empathy Scale), CREA (Creative Intelligence) and a self-concept questionnaire are analyzed. A descriptive analysis of the study variables was carried out using the calculation of the Mean and Standard Deviation. Subsequently, the correlations between variables were calculated using the Pearson correlation index. Finally, for the comparison between groups, a one-way ANOVA was performed. Among the results were relationships between performance, skills and reading comprehension, between the qualification of the subject of Mathematics and the level of cognitive empathy, and self-concept, and between creativity and mathematical proficiency and critical reading comprehension. When comparing students with medium and high performance, differences were found in their level of reading comprehension and self-concept. Students with low and high creativity differ in their level of mathematical proficiency. It is latent the need to design neuropsychological intervention programs that work together on all these variables, in order to achieve an integral development of our students, both in cognitive, emotional and social aspects.

\section{Palabras clave / Keywords}

rendimiento académico, competencias, comprensión lectora, empatía, creatividad, autoconcepto, Educación Primaria academic performance, competences, reading comprehension, empathy, creativity, self-concept, Primary Education. 


\section{Introducción}

La Educación y la neurociencia se han ido desarrollando en estrecha colaboración y se pueden apoyar mutuamente para la mejora educativa por su utilización en los procesos de enseñanza-aprendizaje en la práctica educativa. La necesaria relación y la utilización en Educación de la neuropsicología consigue un avance que favorece el conocimiento, no solo de la localización de las funciones cerebrales, sino también la complejidad del procesamiento de la información en el rendimiento académico, las competencias básicas, la comprensión lectora, la empatía, la creatividad y el autoconcepto, que requieren de una sincronización armónica de múltiples redes neuronales.

El acceso a esta información por parte de los educadores está haciendo posible la aplicación de la neuropsicología en el ámbito educativo, de forma que se puede acceder a recientes estudios y publicaciones, como los de Martín-Lobo (2015), que muestran programas con orientación neuropsicológica para optimizar el rendimiento integral de los alumnos. Así, algunos ejemplos de este tipo de programas son los realizados por autores como: García y Llamas (2015) para el desarrollo de las inteligencias múltiples, López (2015) para favorecer la creatividad, Pradas y de la Peña (2015) para mejorar las dificultades de lenguaje o Martínez (2015) para el fomento de la lectura.

El inicio de esta investigación parte de la resolución de 20 de mayo de 2016, de la Dirección General de Política Educativa Escolar, por la que se convoca la evaluación individualizada de tercer curso de Educación Primaria en el curso escolar 2015-2016. La Ley Orgánica 2/2006, de 3 de mayo, de Educación, modificada por la Ley Orgánica 8/2013, de 9 de diciembre, para la Mejora de la Calidad Educativa, en el artículo 20.3 determina que los centros docentes realizarán una evaluación individualizada a todos los alumnos y alumnas al finalizar el tercer curso de Educación Primaria, según dispongan las Administraciones Educativas. Por todo ello, se convoca a todo el alumnado escolarizado en ese curso a la realización de dichas pruebas celebradas el día 31 de mayo de 2016.

El término competencias en el sistema educativo, en los últimos años, ha adquirido una gran relevancia por la obligatoriedad de su inclusión en las distintas áreas educativas, diferenciándose del concepto de objetivo y entendiéndose como las capacidades a desarrollar por los alumnos.

La evolución del término competencia en el sentido histórico más utilizado, comienza en el mundo laboral y profesional, de ahí que sea desde este campo del que proceden la mayoría de las definiciones (Colás, 2005). Por tanto, el concepto de competencia surge, inicialmente, destinado para el desarrollo laboral sustituyendo al término cualificación (Álvarez Morán, Pérez Collera y Suárez Álvarez (2008)). En consecuencia, una competencia necesita de un conocimiento sobre lo que se basa la actuación concreta o el desempeño (González Jiménez et al., 2010), un saber-actuar que es más que un saberhacer (Skill). En este sentido, skill significa destreza, aptitud, agilidad y habilidad, por lo que es posible, y más apropiado, relacionar la competencia con poder-actuar (Lenoir y Morales-Gómez, 2011).

En la línea de cómo se entiende el término de competencia, en el informe Delors (1996), se plantea este concepto referido al aprendizaje a lo largo de la vida, sostenido en los cuatro pilares de la Educación: Aprender a conocer, aprender a hacer, aprender a vivir juntos y aprender a ser.

El Capítulo V del Boletín Oficial de Castilla y León, núm. 117 viernes, 20 de junio de 2014, p. 44222, indica la importancia de la Educación Primaria porque con ella se inicia la escolarización obligatoria y se ponen las bases en las que asentará todo aprendizaje posterior. El alumnado, al finalizar dicha etapa, debe disponer de los conocimientos, competencias y habilidades básicas, hábitos de trabajo individual y de equipo, de responsabilidad en el estudio, así como actitudes de confianza en sí mismos, interés, curiosidad, sentido crítico y creatividad en el aprendizaje y la iniciativa emprendedora, que le permitan proseguir con éxito su formación en la Educación Secundaria Obligatoria.

Base de esta investigación es el artículo 31 (Boletín Oficial de Castilla y León, núm. 117 viernes, 20 de junio de 2014, p. 44222), donde aparecen las evaluaciones individualizadas. Los centros docentes realizarán una evaluación individualizada al alumnado al finalizar el tercer curso de Educación Primaria, según disponga la consejería competente en materia educativa. Esta evaluación comprueba el grado de dominio de las destrezas, capacidades y habilidades en expresión y comprensión oral y escrita, cálculo y resolución de problemas en relación con el grado de adquisición de la competencia lingüística y matemática.

La Educación, por lo tanto, ha de contemplar las competencias matemática y lingüística en la formación de los alumnos, pero también es importante que se tengan en cuenta otras habilidades de gran importancia para el desarrollo íntegro de los alumnos, tales como la comprensión lectora, la empatía, la creatividad y el autoconcepto.

Destacamos, dentro de este enfoque neuropsicológico, la implicación de las áreas cerebrales en procesos como los que se presentan a continuación: en cuanto a la competencia matemática, para el cálculo exacto en operaciones encontramos estructuras implicadas como el giro angular izquierdo, 
para la realización de un cálculo aproximado es necesaria la activación bilateral del surco intraparietal, para llevar a cabo estrategias y técnicas complejas deben ponerse en funcionamiento áreas del surco intraparietal izquierdo y otras del lóbulo parietal, del frontal inferior, giro fusiforme... Para el desarrollo de la competencia lingüística destacamos el área de Wernike y Broca. Es fundamental mencionar además las neuronas espejo de las áreas promotoras, envueltas en el proceso de la empatía, estas áreas se creía que estaban únicamente implicadas en el reconocimiento de una acción determinada, pero podemos encontrarlas involucradas en la comprensión de la conducta respecto a los demás (Jacoboni, et al., 1999; Tettamanti, et al., 2005). Otras estructuras cerebrales relevantes son el lóbulo frontal, encargado de las emociones, o el córtex prefrontal y el hipocampo, relacionados con el autoconcepto y autoestima. La creatividad se encuentra asociada al funcionamiento de diferentes partes del cerebro. Por tanto, sus funciones cognitivas no están localizadas en un área cerebral específica, sino que se basan en el funcionamiento de complejos sistemas funcionales entre estructuras (Lee, Harrison y Mechelli, 2003): hemisferio derecho, córtex prefrontal, relacionadas con conexiones cortico-corticales y cortico-subcorticales, y lóbulos temporales.

Cabe destacar el concepto de comprensión lectora como el proceso de construcción de significado personal de un texto mediante una interacción activa (Catalá, Catalá, Molina y Monclús, 2007), como el razonamiento verbal que mide la capacidad de entendimiento y de crítica sobre el contenido de la lectura (Cooper, 1990). Para ello es fundamental destacar las estrategias de comprensión lectora, que son un conjunto de acciones ordenadas y finalizadas que deben adquirirse, siempre dirigidas a la consecución de una meta (Solé, 2001). Estas estrategias de enseñanza, entendidas como el conjunto de decisiones, son tomadas en el proceso de enseñanza-aprendizaje para orientar dicho proceso con el fin de promover la generación de conocimiento en los alumnos (Anijovich y Mora, 2009), enfocado siempre en cómo enseñar un contenido, por qué y para qué.

Otro componente a tener en cuenta en el desarrollo de una persona es la empatía. La importancia de esta variable radica en que favorece la conducta orientada hacia el bienestar y, además, nuevamente, la resolución positiva de problemas (Álvarez, Carrasco y Fustos, 2010). Estructuralmente, la empatía posee dos componentes, uno cognitivo y otro emocional (Jolliffe y Farrington, 2006).

Como hemos reflejado, otro de los aspectos de gran relevancia en la educación es la creatividad. Esta variable puede definirse de muchas maneras, pero una de las más aceptadas es la que la concibe como un atributo que todas las personas tienen. De hecho, Simonton (2000) no duda en plantear que la creatividad es una característica que implica connotaciones positivas: "la creatividad es un buen atributo que la gente posee" (p. 151). Otros autores, como Carrascal y Solera (2014), destacan que la creatividad estaría relacionada con la capacidad de generar ideas o resolver problemas. Y no cabe duda de que, en la vida de las personas, tener desarrollada la capacidad de resolución de problemas es un aspecto importante.

Otra característica de gran relevancia, como se ha indicado con anterioridad, en el desarrollo de las personas y que se ha demostrado que influye en la adaptación escolar, es el autoconcepto (Rodríguez Fernández, Ramos-Díaz, Ros y Zuazagoitia, 2018). Este aspecto puede ser definido como aquellas percepciones que una persona tiene sobre ella misma según la valoración personal y la evaluación de los otros (Shavelson, Hubner, y Stanton, 1976).

Existen diversos trabajos que ponen de manifiesto la necesidad de trabajar en el campo educativo todas las variables de estudio destacadas, ya que son la base del desarrollo de conocimiento en las aulas hoy en día, fundamentales para implementarlas en el campo educativo. La competencia lectora es destacada como una de las herramientas psicológicas más relevantes en los procesos de enseñanza-aprendizaje, ya que su carácter transversal va unido al desarrollo de competencias, y conlleva efectos colaterales positivos o negativos sobre el resto de áreas académicas (GutiérrezBraojos y Salmerón Pérez, 2012), por todo ello en los últimos años se ha enfatizado el papel de las estrategias de aprendizaje, como herramientas psicológicas que facilitan a los estudiantes el proceso transaccional lector. Otros autores como Franco (2009), analizan la importancia del autoconcepto y la creatividad en la población infantil para que la expresión de la capacidad creativa se relacione de forma positiva en la manifestación del autoconcepto. Por otra parte, estudios sobre la empatía son fundamentales para su desarrollo en estas edades tempranas ya que recientes investigaciones indican la importancia del término en Educación y su relevancia en la implicación del proceso educativo y el rendimiento académico. Autores como Decety e Ickes (2009) investigan sobre la importancia de las cualidades empáticas y el comportamiento de los alumnos en términos sociales y su relación con el logro académico en el centro educativo. Por todo ello, identificamos estas variables para su análisis ya que, como indican Garaigordobil y Berrueco (2007), el concepto de los alumnos de Educación Infantil es un factor preventivo, al igual que ocurre con los niveles de creatividad, la comprensión lectora, competencias, los aspectos socioemocionales, como la empatía, y son, por lo tanto, elementos 
claves en el diseño de programas de intervención. Con este estudio se pretende relacionar todas las variables indicadas para optimizar el proceso de enseñanza-aprendizaje de los estudiantes.

\section{Método}

\subsection{Objetivos e hipótesis}

El objetivo general de esta investigación es el estudio de la relación e influencia entre las variables neurocognitivas y socioemocionales implicadas en el proceso de enseñanza-aprendizaje, y se subdivide en:

- Estudiar el nivel de rendimiento, competencias lingüística y matemática, comprensión lectora, empatía, creatividad y autoconcepto de los alumnos de tercero de Educación Primaria.

- Analizar la relación entre las diferentes variables de estudio.

- Comparar los alumnos con diferente nivel de rendimiento académico y de creatividad en cuanto a sus competencias matemática y lingüística y su nivel de comprensión lectora, empatía y autoconcepto.

Como resultado de los objetivos propuestos, las hipótesis planteadas son:

- Los alumnos obtendrán niveles medio-altos en las variables evaluadas.

- Existirá una relación entre las variables de naturaleza neurocognitiva (calificaciones de las asignaturas, competencias lingüística y matemática, y comprensión lectora) y las variables de naturaleza socioemocional (empatía, creatividad y autoconcepto).

- Se hallarán diferencias en función del rendimiento académico y de la creatividad. Los alumnos con calificaciones más elevadas y alta creatividad puntuarán también más alto en el resto de variables evaluadas.

\subsection{Muestra}

En este estudio se seleccionaron por muestreo aleatorio a 82 alumnos/as, 42 niños y 40 niñas, de tercero de Educación Primaria de un colegio concertado de Ávila (España). Todos ellos participaron voluntariamente y con autorización de los padres. Ninguno de los participantes del estudio era Alumno con Necesidades Específicas de Apoyo Educativo (ACNEAE).

\subsection{Instrumentos y materiales}

\section{Rendimiento académico}

Con el fin de evaluar el rendimiento se solicitaron las calificaciones de la evaluación final del curso 2016-2017 obtenidas por los alumnos en cada una de las asignaturas cursadas. Se calculó la media para obtener el rendimiento global.

Prueba de evaluación individualizada

Esta prueba se compone de pruebas orales y escritas que evalúan las competencias lingüística y matemática (ver subpruebas y temporalización en la Tabla 1). Por un lado, la competencia lingüística se calcula a partir de la media de las puntuaciones obtenidas en el apartado de comprensión oral y escrita (50\%) y de expresión escrita (50\%). Por otro lado, la competencia matemática se computa como la media de los resultados en cálculo: números y operaciones $(40 \%)$ y en resolución de problemas $(60 \%)$. La calificación global es la media de las calificaciones en la competencia lingüística en castellano y la matemática. 


\begin{tabular}{|c|c|c|}
\hline Fecha y horario & Procedimiento & Tiempo \\
\hline 31 de mayo/09:00h & $\begin{array}{l}\text { Preparación del aula/comprobación de material/asignación } \\
\text { de códigos de identificación al alumnado }\end{array}$ & 30 \\
\hline 31 de mayo/09:30h & $\begin{array}{l}\text { Distribución pruebas de competencia } \\
\text { matemática/Explicación de instrucciones }\end{array}$ & $10^{\prime}$ \\
\hline 31 de mayo/09:40h & Realización pruebas de competencia matemática & 50 \\
\hline 31 de mayo/10:30h & Descanso & 30 \\
\hline 31 de mayo/11:05h & $\begin{array}{l}\text { Distribución pruebas de competencia lingüística/Explicación } \\
\text { de instrucciones }\end{array}$ & $10^{\prime}$ \\
\hline 31 de mayo/11:15h & Realización pruebas de competencia lingüística & $55^{\prime}$ \\
\hline
\end{tabular}

Fuente: Elaboración propia

Prueba ACL: Evaluación de la comprensión lectora

La prueba ACL (Catalá, Catalá, Molina Monclús, 2007) consta de textos adaptados a alumnos de Primaria. En concreto, ACL-3 está dirigido a estudiantes de tercero y consta de siete textos con 25 ítems para responder, además de un ejemplo de texto con preguntas para responder colectivamente y entrenar a los alumnos. La puntuación directa se obtiene de la suma de un punto por cada ítem correctamente contestado (hasta un máximo de 25 puntos), a mayor puntuación mayor comprensión lectora. A partir de dicha puntuación, la prueba incluye un baremo basado en la edad para transformarlas en puntuaciones decatipo, con un rango de valores comprendido entre 0 y 10 . La escala permite la obtención de una puntuación en comprensión literal (puntuación máxima 9), reorganización (puntuación máxima 5), comprensión inferencial (puntuación máxima 8) y comprensión crítica (puntuación máxima 3). La fiabilidad del instrumento, calculada a través del índice Alfa de Cronbach es .71. Para la muestra del estudio, la prueba presenta una fiabilidad de .81 .

\section{BES-A: Escala de Empatía Básica Abreviada}

La prueba $B E S-A$ es una versión abreviada de 9 ítems (derivados del estudio piloto de Oliva et al., 2011) de la Escala de Empatía Básica (Jollife y Farrington, 2006). Los ítems de esta escala se agrupan en dos subescalas: empatía afectiva (puntuación máxima 20) y empatía cognitiva (puntuación máxima 25). La puntuación de cada escala se obtiene de la suma de los ítems. Esta adaptación se administra en 5 minutos y está ponderado en percentiles por edad y sexo. La fiabilidad del instrumento, calculada a través del índice Alfa de Cronbach es superior a .70. En concreto, para la muestra del estudio, este instrumento presenta una fiabilidad de .73 .

\section{CREA: Inteligencia creativa}

El test de Inteligencia Creativa (CREA) permite una valoración cognitiva de la creatividad (Corbalán et al., 2003). Esta prueba es una medida de la creatividad a través de la capacidad de los sujetos para generar preguntas sobre un dibujo durante un tiempo limitado. Cada pregunta formulada por los sujetos presume la activación de un nuevo esquema cognitivo. La prueba cuenta con tres dibujos diferentes: A y $B$, para sujetos adolescentes y adultos; y C, para niños. El tiempo de aplicación de la prueba es de cuatro minutos. La puntuación directa se transforma en puntuación centil que es interpretada según el baremo de la prueba como creatividad baja (1 a 25), media (26 a 74) o alta (75 a 99). La fiabilidad, analizada mediante formas paralelas, alcanza un $\alpha=.87$. Más concretamente, la fiabilidad alcanzada para la muestra del estudio es de .50 .

\section{Cuestionario de autoconcepto}

El cuestionario de autoconcepto se elabora para este estudio y consta de cinco ítems, a los que el estudiante debe contestar en función del nivel de acuerdo con el mismo en una escala Likert, donde se establecen los siguientes niveles: $A$ (muy en desacuerdo), $B$ (en desacuerdo), $C$ (de acuerdo) y $D$ (muy de acuerdo). La fiabilidad del instrumento, calculada a través del índice Alfa de Cronbach es .62. El rango de puntuaciones que puede obtenerse en la prueba oscila entre 1 y 20 puntos.

1. En conjunto, me siento satisfecho conmigo mismo.

2. Pienso que soy un chico/a listo/a.

3. Creo que tengo un buen número de buenas cualidades.

4. Muchos de mis compañeros dicen que soy bueno en los estudios.

5. Mis padres están contentos con mis notas.

$\begin{array}{llll}A & B & C & D \\ A & B & C & D \\ A & B & C & D \\ A & B & C & D \\ A & B & C & D\end{array}$




\subsection{Procedimiento}

En primer lugar, se solicitó la autorización al equipo directivo del centro para llevar a cabo el estudio. Tras este paso, se procedió a pedir consentimiento por escrito a los padres de los alumnos. Posteriormente, se coordinaron los horarios y grupos para la realización de las pruebas. El orden de realización fue el siguiente: En primer lugar, la prueba de evaluación individualizada (consultar procedimiento y temporalización en la Tabla 1); en segundo lugar, la prueba de comprensión lectora (ACL) en una sesión de 45 minutos; en tercer lugar, el cuestionario de empatía (BES-A), el test de Creatividad (CREA-C) y el cuestionario de autoconcepto, las tres administradas en una misma sesión de 45 minutos. Todos los instrumentos fueron aplicados de manera colectiva y en el aula habitual de cada alumno.

\subsection{Análisis de datos}

Con el fin de dar respuesta a los objetivos, en primer lugar, se realizó un análisis descriptivo de las variables de estudio recurriendo al cálculo de la media y la desviación típica. Posteriormente, se calcularon las correlaciones entre variables a través del índice de correlación de Pearson. Finalmente, para la comparación entre grupos se realizaron ANOVA de un factor.

\section{Resultados}

A continuación, se presentan los resultados obtenidos a partir de los análisis de los datos recogidos.

\subsection{Análisis descriptivo}

A nivel descriptivo, se pueden observar en la Tabla 2, los valores promedio y las desviaciones típicas de las variables: calificaciones en lengua, matemáticas y total, resultados en la competencia lingüística, matemática y la media de ambas, comprensión lectora, empatía, creatividad y autoconcepto. Las puntuaciones obtenidas ponen de manifiesto un adecuado rendimiento académico dado que la media en todas las variables de naturaleza cognitiva es superior a 7.

\section{Tabla 2}

Media y Desviación Típica de las variables

\begin{tabular}{llll}
\hline Variable (rango puntuación) & $\mathbf{N}$ & $\boldsymbol{M}$ & $\boldsymbol{D T}$ \\
\hline Nota media & 82 & 7.62 & 1.15 \\
Calificación en lengua & 82 & 7.30 & 1.55 \\
Calificación en matemáticas & 82 & 7.52 & 1.51 \\
Competencia lingüística & 82 & 8.26 & 0.86 \\
Competencia matemática & 82 & 7.22 & 1.78 \\
Competencia media & 82 & 7.71 & 1.15 \\
ACL (0-25) & 82 & 14.56 & 4.47 \\
C. Literal (0-9) & 82 & 5.32 & 1.83 \\
Reorganización (0-5) & 82 & 2.91 & 1.14 \\
C. Inferencial (0-8) & 82 & 4.84 & 1.71 \\
C. Crítica (0-3) & 82 & 1.52 & 0.91 \\
BES afectiva(0-20) & 82 & 12.68 & 3.45 \\
BES cognitiva (0-25) & 82 & 19.91 & 3.19 \\
BES total (0-45) & 82 & 32.72 & 5.46 \\
CREA & 82 & 8.18 & 2.99 \\
Autoconcepto (0-20) & 82 & 16.82 & 2.54 \\
\hline
\end{tabular}

Fuente: Elaboración propia

Los resultados obtenidos por la muestra en rendimiento académico (calificaciones) y competencias ponen de manifiesto niveles medio-altos. Respecto a comprensión lectora, los alumnos consiguen un nivel medio en general. En empatía y autoconcepto se observa un nivel medio-alto. Por el contrario, los resultados en el test CREA ponen de manifiesto niveles medio-bajos de creatividad.

\subsection{Análisis correlacional}


En cuanto a las correlaciones realizadas, en la Tabla 3 se muestran los resultados obtenidos respecto a las relaciones entre las puntuaciones obtenidas por los alumnos en las diferentes pruebas administradas y sus calificaciones.

A la vista de la Tabla 3, se aprecia una correlación significativa entre las notas, las competencias y la comprensión lectora. Adicionalmente, la nota de matemáticas correlaciona con el nivel de empatía cognitiva y el autoconcepto de los alumnos. En cuanto a la creatividad, se detecta que esta correlaciona positivamente con la competencia matemática, así como con la comprensión lectora crítica. 


\section{PREPRINT}

Tabla 3

Correlaciones entre estrategias de aprendizaje y calificaciones

\begin{tabular}{|c|c|c|c|c|c|c|c|c|c|c|c|c|c|c|c|c|}
\hline Variable & 1 & 2 & 3 & 4 & 5 & 6 & 7 & 8 & 9 & 10 & 11 & 12 & 13 & 14 & 15 & 16 \\
\hline 1.Nota media & 1 & $.93^{\star \star}$ & $.88^{\star \star}$ & .05 & .19 & .14 & .13 & $.46^{\star \star}$ & $.60^{\star \star}$ & $.61^{\star \star}$ & $.69^{\star \star}$ & $.59^{\star \star}$ & $.47^{\star \star}$ & $.61^{\star \star}$ & $.48^{\star \star}$ & .22(.05) \\
\hline 2.Nota lenqua & & 1 & $.84^{\star \star}$ & .03 & 17 & 12 & 13 & $47^{\star \star}$ & $.57^{\star \star}$ & $.61^{\star \star}$ & $.69^{\star \star}$ & $.58^{\star \star}$ & $46^{\star \star}$ & $.63^{\star \star}$ & $49^{\star \star}$ & 20 \\
\hline 3.Nota matemáticas & & & 1 & -.03 & $.23^{\star *}$ & .11 & .03 & $.44^{\star *}$ & $.59^{\star \star}$ & $.62^{\star *}$ & $.61^{\star *}$ & $.54^{\star \star}$ & $.39^{\star \star}$ & $.53^{\star \star}$ & $.46^{\star *}$ & $.24^{*}$ \\
\hline 4.BES afectiva & & & & 1 & $.31^{* *}$ & $.83^{\star *}$ & -.04 & .01 & .06 & .05 & .06 & .11 & -.02 & .01 & .06 & -.06 \\
\hline 5.BES cognitiva & & & & & 1 & $.75^{\star \star}$ & .20 & -.02 & .10 & .07 & .11 & .15 & .03 & .05 & .10 & .09 \\
\hline 6.BES total & & & & & & 1 & .07 & -.04 & .10 & .06 & .10 & .18 & .01 & .02 & .08 & -.01 \\
\hline 7.CREA & & & & & & & 1 & -.11 & $.23^{\star}$ & .09 & .17 & .15 & .02 & .15 & $.24^{\star}$ & .06 \\
\hline 8.Competencia & & & & & & & & 1 & $.25^{\star}$ & $.58^{\star \star}$ & $.49^{\star *}$ & $.34^{\star *}$ & $.35^{\star \star}$ & $.52^{\star *}$ & $.33^{\star \star}$ & .18 \\
\hline lingüística & & & & & & & & & & & & & & & & \\
\hline $\begin{array}{l}\text { 9.Competencia } \\
\text { matemática }\end{array}$ & & & & & & & & & 1 & $.88^{\star \star}$ & $.52^{\star \star}$ & $.46^{\star \star}$ & $.24^{\star \star}$ & $.45^{\star *}$ & $.48^{\star \star}$ & .05 \\
\hline 10.Competencia media & & & & & & & & & & 1 & $.58^{\star \star}$ & $.48^{\star *}$ & $.32^{\star \star}$ & $.55^{\star *}$ & $.48^{\star \star}$ & .07 \\
\hline 11.ACL & & & & & & & & & & & 1 & $.89^{* *}$ & $.72^{\star \star}$ & $.88^{* *}$ & $.60^{\star \star}$ & .11 \\
\hline 12.C. Literal & & & & & & & & & & & & 1 & $.57^{\star \star}$ & $.65^{\star \star}$ & $.49^{\star \star}$ & .04 \\
\hline 13.Reorganización & & & & & & & & & & & & & 1 & $.65^{\star \star}$ & $.49^{\star \star}$ & .06 \\
\hline 14.C. Inferencial & & & & & & & & & & & & & & 1 & $.56^{\star \star}$ & .15 \\
\hline 15.C. Crítica & & & & & & & & & & & & & & & 1 & .11 \\
\hline 16.Autoconcepto & & & & & & & & & & & & & & & & 1 \\
\hline
\end{tabular}

Fuente: Elaboración propia 


\subsection{Análisis de comparación}

En primer lugar, con el fin de evaluar si existían diferencias significativas entre los alumnos con rendimiento medio-alto, se dividió a la muestra en función de sus calificaciones. En la Tabla 4 se pueden observar las medias y desviaciones típicas de las puntuaciones de cada grupo en el rendimiento.

Tabla 4

Estadísticos descriptivos en las diferentes variables en función del rendimiento

\begin{tabular}{lllll}
\hline Variable & \multicolumn{2}{c}{ Rendimiento medio } & \multicolumn{2}{c}{ Rendimiento alto } \\
\hline & $M$ & $D T$ & $M$ & $D T$ \\
ACL & 12.03 & 3.79 & 16.64 & 3.90 \\
BES-A & 32.24 & 6.24 & 33.11 & 4.76 \\
CREA & 8.03 & 2.90 & 8.31 & 3.08 \\
Autoconcepto & 16.24 & 2.77 & 17.29 & 2.25 \\
\hline
\end{tabular}

Fuente: Elaboración propia

Como se detalla en la tabla anterior, el grupo con alto rendimiento puntúa más alto en todas las variables evaluadas en comparación con el grupo de bajo rendimiento.

A continuación (Tabla 5) se detallan los resultados obtenidos en cuanto a la comparación de los grupos de rendimiento, tras la comprobación del supuesto de homogeneidad de varianzas entre los grupos en las diferentes variables, en su nivel de comprensión lectora, empatía, creatividad y autoconcepto.

Tabla 5

Resultados ANOVA comparando medias de grupos de rendimiento medio-alto

\begin{tabular}{llll}
\hline Variables & $\boldsymbol{F}$ & $\boldsymbol{p}$ & $\eta \mathbf{2}$ \\
\hline ACL & 29.22 & .00 & .27 \\
BES-A & .51 & .48 & - \\
CREA & .18 & .67 & - \\
Autoconcepto & 3.75 & .04 & .31 \\
\hline
\end{tabular}

Fuente: Elaboración propia

Como puede verse, los alumnos con medio y alto rendimiento difieren en las puntuaciones obtenidas en la comprensión lectora $(F=29.22 ; p<.01 ; \eta 2=.27)$ y el cuestionario de autoconcepto $(F=3.75 ; p<.05$; $\eta_{2}=.31$ ). En ambos casos, y a partir de la interpretación del tamaño del efecto alcanzado, vemos que la influencia de la variable independiente sobre la dependiente es media.

Tras el análisis en función del rendimiento, y con el objetivo de evaluar si existían diferencias significativas entre los alumnos con creatividad baja-alta, se dividió a la muestra en función de sus puntuaciones en el test CREA. En la Tabla 6 se pueden observar las medias y desviaciones típicas de las puntuaciones de cada grupo en función del nivel de creatividad.

Tabla 6

Estadísticos descriptivos en las diferentes variables en función de la creatividad

\begin{tabular}{lcccc}
\hline Variable & \multicolumn{3}{c}{ Creatividad baja } & \multicolumn{2}{c}{ Creatividad alta } \\
\hline & $M$ & $D T$ & $M$ & $D T$ \\
Nota media & 7.56 & 1.16 & 7.86 & 1.12 \\
Nota lengua & 7.23 & 1.56 & 7.59 & 1.12 \\
Nota matemáticas & 7.48 & 1.55 & 7.71 & 1.36 \\
Competencia lingüística & 8.32 & .81 & 8.02 & 1.05 \\
Competencia matemática & 6.97 & 1.80 & 8.19 & 1.39 \\
Competencia media & 7.62 & 1.14 & 8.06 & 1.14 \\
ACL (0-25) & 14.43 & 4.26 & 15.06 & 5.30 \\
BES- $A$ & 32.49 & 5.52 & 33.59 & 5.28 \\
Autoconcepto & 16.86 & 2.59 & 16.65 & 2.40 \\
\hline
\end{tabular}

Fuente: Elaboración propia

A nivel descriptivo, se puede observar que los alumnos con alta creatividad puntúan más alto en todas las variables, a excepción de la competencia lingüística y el autoconcepto, en comparación con el grupo de baja creatividad (ver Tabla 6).

En la Tabla 7 se detallan los resultados obtenidos en cuanto a la comparación de los grupos con creatividad baja y alta en su nivel de rendimiento, competencias, comprensión lectora, empatía y 
autoconocimiento (previamente, se comprobó que se cumplía en todos los casos el supuesto de homogeneidad de varianzas).

Tabla 7

Resultados ANOVA comparando medias de grupos de creatividad baja-alta

\begin{tabular}{llll}
\hline Variables & $\boldsymbol{F}$ & $\boldsymbol{p}$ & $\boldsymbol{\eta 2}$ \\
\hline Nota media & .91 & .34 & - \\
Nota lengua & .71 & .40 & - \\
Nota matemáticas & .71 & .58 & - \\
Competencia lingüística & 1.61 & .21 & - \\
Competencia matemática & 6.79 & .01 & .40 \\
Competencia media & 2.03 & .16 & - \\
ACL (0-25) & .26 & .61 & - \\
BES-A & .54 & .46 & - \\
Autoconcepto & .10 & .76 & - \\
\hline
\end{tabular}

Fuente: Elaboración propia

Los grupos de nivel bajo y alto de creatividad difieren en la competencia matemática, siendo el tamaño del efecto medio $\left(F=6.79 ; p<.05 ; \eta_{2}=.40\right)$, pero no en el resto de variables analizadas.

En síntesis, los resultados obtenidos son: a nivel descriptivo, los alumnos del estudio presentaron niveles medio-altos en rendimiento académico, competencias lingüística y matemática, comprensión lectora y empatía, niveles medios en autoconcepto y medio-bajos en creatividad. Respecto al análisis correlacional, existe una relación, en primer lugar, entre las notas, las competencias y la comprensión lectora; en segundo lugar, entre la nota de matemáticas y el nivel de empatía cognitiva y el autoconcepto; $y$, en tercer lugar, entre la creatividad y la competencia matemática y la comprensión lectora crítica. La comparación entre grupos arrojó diferencias entre alumnos de medio y bajo rendimiento en sus niveles de comprensión lectora y de autoconcepto y entre alumnos con baja y alta creatividad en su competencia matemática.

\section{Discusión y Conclusiones}

El estudio aquí presentado ha tenido como objetivo estudiar el nivel de rendimiento, competencias lingüística y matemática, comprensión lectora, empatía, creatividad y autoconcepto de los alumnos de tercero de Educación Primaria, con el fin de analizar la relación entre las diferentes variables neuropsicológicas de estudio.

Respecto a los resultados descriptivos encontrados, muestran que el rendimiento obtenido por la muestra de estudio en las pruebas de evaluación individualizada se sitúa en un nivel medio-alto en sus competencias y, por tanto, se puede afirmar que son competentes lingüística y matemáticamente Por otra parte, en cuanto a la comprensión lectora y sus dimensiones, evaluada a través de la prueba ACL (Catalá et al., 2007), como se aprecia en los resultados descriptivos, la muestra en su conjunto se situaría en un nivel medio, correspondiente a un decatipo 6 , tanto en la comprensión global como en los componentes evaluados: comprensión literal, reorganización, comprensión inferencial y comprensión crítica. Los alumnos son capaces de entender diferentes tipos de textos y analizarlos, tanto de manera literal, como a nivel de organización de datos, de realización de inferencias más allá de la información ofrecida y razonar críticamente los contenidos. Tal y como marca el currículo establecido para el nivel educativo de tercero de Primaria estos aspectos son esenciales y deben ser trabajados dentro de la competencia lingüística y de manera transversal en todas las asignaturas dada su repercusión académica a nivel global (Gutiérrez-Braojos y Salmerón, 2012).

Respecto a los resultados en la variable empatía, evaluada a través del test BES (Jollife y Farrington, 2006) en versión abreviada, se aprecia que la muestra obtiene niveles medio-altos en dicha variable. El hecho de encontrarse con puntuaciones medias-altas en esta variable va en la línea de determinados estudios que señalan que la empatía se adquiere gradualmente conforme avanza la edad de las personas, sobre todo en la toma de perspectiva, fantasía y la preocupación empática (Retuerto, 2004). Aunque este estudio solo analizó la empatía cognitiva, afectiva y total, los valores medio-altos señalan que emocional y cognitivamente los alumnos van desarrollando las habilidades que permiten procesar dicha información.

En relación a la puntuación obtenida por la muestra en la prueba CREA (Corbarán et al., 2003) con una puntuación media de 8.18 ( $D T=2.99)$, situando a la muestra en el centil 55 , lo que sitúa a la muestra en una ejecución media. Este resultado indicaría que los participantes no muestran una ejecución más 
destacada en creatividad quizás por exhibir una cognición más centrada en estrategias convergentes (Corbarán et al., 2003).

Respecto a la variable autoconcepto, evaluada con un cuestionario elaborado para el estudio y con una fiabilidad Alfa de Cronbach aceptable $(\alpha=.62)$, la muestra obtiene una puntuación de 16.82 $(D T=2.52)$, por lo que se sitúa en un nivel por encima de la media, ya que el rango de puntuaciones oscila entre 1 y 20 puntos, por lo que puede afirmarse que, en general, la muestra tiene un autoconcepto superior a la puntuación media de la prueba.

En cuanto a los resultados correlacionales, como puede verse en el apartado de resultados, las puntuaciones obtenidas a través de la evaluación individualizada, a saber, competencias lingüística, matemática y media, correlacionan positivamente con algunas de las variables analizadas. La competencia lingüística correlaciona positivamente (aparte de con el resto de las competencias) con el rendimiento académico medio, el rendimiento en las asignaturas de lengua y matemáticas, la comprensión lectora (tanto a nivel general como en comprensión literal, reorganización, comprensión inferencial y comprensión crítica). El hecho de que la competencia lingüística correlacione positivamente con el rendimiento académico de dichas asignaturas y sus competencias asociadas, puede deberse a que el uso de la lengua es imprescindible en el desarrollo del aprendizaje, tanto es así que tal y como señalan Rico y Níkleva (2016) en la vida diaria se debe estar lo suficientemente capacitado para abordar la escritura.

En cuanto a la comprensión lectora y cada uno de sus componentes, la existencia de correlación puede deberse a que la competencia lingüística se calcula a partir de la media ponderada de las puntuaciones obtenidas en el apartado de comprensión oral y escrita (50\%) y de expresión escrita $(50 \%)$. Por lo tanto, el hecho de tener una buena competencia lingüística equivale a tener desarrollada una capacidad para comprender y producir textos en diferentes situaciones que exijan comunicación (Rico y Níkleva, 2016). Sin embargo, a pesar de que otros estudios sí habían encontrado relación entre la comprensión y algunas habilidades sociales y emocionales, así como una incidencia positiva o negativa en la autoestima o el autoconcepto del escolar (Hines, 2009), en este estudio no se ha encontrado explícitamente.

En relación con las correlaciones obtenidas por la competencia matemática, cabe destacar, como se ha indicado anteriormente, que esta se relaciona positivamente con la lingüística y la media, así como la comprensión lectora en cada uno de sus componentes y el rendimiento académico medio y de las asignaturas de matemáticas y lengua. Además, se ha encontrado relación estadísticamente significativa entre la competencia matemática y la creatividad. En este sentido, cabe destacar que dentro del complejo estudio de la creatividad, uno de los enfoques más relevantes ha sido su estudio desde la perspectiva de la solución de problemas. Y, sin duda, las Matemáticas implican resolución de diferentes problemas y desde una perspectiva escolar, es muy importante identificar las estrategias alternativas de resolución y comprensión de los mismos (Sánchez y Fiol, 2016). Concretamente, se han comenzado a realizar estudios que relacionan la creatividad desde el enfoque de resolución de problemas, con la competencia matemática en geometría, en concreto estudiando la fase del insight de dicho proceso creativo (Sánchez y Fiol, 2016).

La creatividad también correlaciona con la comprensión crítica en la muestra de estudio, en el sentido de que, a mayores puntuaciones en creatividad, los alumnos también obtenían resultados superiores en el componente crítico de la comprensión. Estos hallazgos se encuentran en la línea de Baños et al. (2011) que argumentan que manejar aspectos críticos supone desarrollar una capacidad creativa capaz de producir cambios. La creatividad implica el hecho de reformular ideas, y éstas surgen de un componente crítico que posibilita la reformulación. Halpern (1984) defendió que la creatividad puede considerarse como la habilidad que poseemos para crear nuevas ideas y sus combinaciones para cubrir una necesidad, necesidad que surgiría de una crítica ante una situación que no satisface un deseo. Por todo ello, tal y como señala García Restrepo (2014) la Educación en estos tiempos debe contemplar estrategias innovadoras de enseñanza, que sean facilitadoras del pensamiento creativo y crítico.

Dentro del objetivo general del estudio, también se había contemplado comparar a los alumnos con diferente nivel de rendimiento académico y de creatividad en cuanto a sus competencias, comprensión lectora, empatía y autoconcepto. En cuanto a los resultados comparativos al separar a los alumnos en dos grupos, a saber, medio y bajo rendimiento, solo se encontraron diferencias significativas en la comprensión lectora y el autoconcepto. El hecho de haber encontrado disparidades en ambos grupos en función del rendimiento y la comprensión se muestra congruente con la literatura científica que exhibe una estrecha relación entre ambas variables. De hecho, deficiencias en la lectura y comprensión lectora en los estudiantes pueden ser los principales factores que afectan el rendimiento académico (Guzmán, Véliz y Reyes, 2017; Samamé, 2014). Por otra parte, la relación entre el autoconcepto y el 
rendimiento académico no es nueva. Revuelta, Rodríguez, Ormaza y Ramos (2016) analizaron la relación de ambas variables, considerando el autoconcepto como un constructo multidimensional y encontraron que eran los dominios autoconcepto académico y autoconcepto social los que presentan correlaciones más intensas con las calificaciones de las diferentes asignaturas. Por lo que los hallazgos del estudio presentado coinciden con los de la literatura previa.

En cambio, al dividir a la muestra en dos grupos en función de creatividad media o baja, solo se hallaron diferencias estadísticamente significativas en ambos grupos en la variable de competencia matemática. Estos hallazgos refuerzan la asociación entre ambas variables que se encontró en el análisis correlacional, evidenciando que las matemáticas implican resolución de diferentes problemas y que la creatividad también puede entenderse desde un enfoque de proceso, en el cual se diferencian diversas etapas de resolución de un problema, como lo contempló Wallas hace casi un siglo (1926) y reforzaron posteriormente autores como Amabile y Gitomer (1984).

Los resultados que aquí se han encontrado ponen de relieve la necesidad de diseñar programas de intervención neuropsicológica que trabajen de manera conjunta todas estas variables, en aras de lograr un desarrollo integral de nuestros alumnos, tanto en aspectos cognitivos, como emocionales y sociales. Futuras líneas de investigación podrían abordar la relación entre las variables mencionadas y el autoconcepto desde una perspectiva multidimensional, y no unidimensional como se ha abordado aquí. Además, sería realmente interesante analizar el efecto que la aplicación de estos programas pudiese tener sobre las variables y evaluadas y otras, como las inteligencias múltiples, que no se han tenido en cuenta en este trabajo. El hecho de incluir las inteligencias, aportaría un plus a la evaluación individualizada de los alumnos y al posterior ajuste de actividades y programas para ajustarse mejor al alumnado.

Esta investigación posee como principal limitación no haber podido separar a los alumnos en niveles medios, bajos y altos en cada variable, ya que la muestra presentaba solo niveles medios y altos, por lo que no se ha podido comprobar el comportamiento de las variables en los niveles más bajos y si las diferencias (de existir) son o no significativas. Por ello, como línea de investigación futura, se plantean diversos objetivos entre los que se incluye aumentar el tamaño y variedad de la muestra.

\section{Referencias}

Álvarez, P., Carrasco, M. y Fustos, J. (2010). Relación de la empatía y género en la conducta prosocial y agresiva, en adolescentes de distintos tipos de establecimientos educacionales. Revista lberoamericana de Psicología: Ciencia y Tecnología, 3(2), 27-36.

Álvarez Morán, S., Pérez Collera, A., Suárez Álvarez, M느. (2008). Hacia un enfoque de la educación en competencias. Madrid: Conserjería de Educación y Ciencia, Servicio de Evaluación, Calidad y Ordenación Académica.

Amabile, T. M., \& Gitomer, J. (1984). Children's Artistic Creativity Effects of Choice in Task Materials. Personality and Social Psychology Bulletin, 10(2), 209-215.

Anijovich, R y Mora, I (2009): Estrategias de enseñanza. Otra mirada al quehacer en el aula. Buenos Aires: AIQUE Educación.

Baños J., Vázquez, M. T., Juárez, A., Molina, E., Rodríguez, M., y Pérez M. (2011). Relevancia de la profesión docente en la escuela del nuevo milenio. Curso básico de formación continua para Maestros en Servicio. México: Secretaría de Educación Pública

Boletín Oficial de Castilla y León, 117/2014, de 20 de junio de 2014

Carrascal, S., y Solera, E. (2014). Creatividad y desarrollo cognitivo en personas mayores. Arte, Individuo y Sociedad, 26(1), 9-19.

Catalá, G., Catalá, M., Molina E., y Monclús, R. (2007). Evaluación de la comprensión lectora. Pruebas ACL. 3aㅡ ed. Barcelona: Graó

Colás Bravo, M. P. (2005). La formación universitaria en base a competencias. En: P. Colas y J. De Pablos (Coord.). La Universidad en la Unión Europea. El Espacio Europeo de Educación Superior y su impacto en la docencia. (pp. 101-123). Málaga: Aljibe.

Cooper, J. D. (1990): Cómo mejorar la comprensión lectora. Madrid: Aprendizaje/Visor/MEC

Corbalán, F. J., Martínez, F., Donolo, D., Alonso, C., Tejerina, M. y Limiñana, R. M. (2003). CREA. Inteligencia Creativa. Madrid: TEA Ediciones.

Decenty J. \& Ickes, W. (2009). The social neuroscience of empathy. Cambridge, Massachussetts: Bradford book MIT Press

Delors, J. (1996). La educación encierra un tesoro. Informe a la Unesco de la comisión internacional sobre la educación para el siglo XXI. Madrid: Santillana/UNESCO.

Franco Justo, C. (2009): Relación entre las variables autoconcepto y creatividad en una muestra de alumnos de educación infantil. Revista electrónica de investigación educativa (REDIE), 8 (1).

Garaigordobil, M. \& Berrueco, L. (2007). Self-concept in 5-year-old children: Relationships with intelligence, neuropsychological maturity, creativity, altruism and empathy. Infancia y Aprendizaje, 30(4):551-564. 
García, M.L. y Llamas, F. (2015). Procesos y Programas de Inteligencias Múltiples. En P. Martín Lobo. Procesos y programas de neuropsicología educativa (pp. 123-138). Madrid: Ministerio de Educación, Cultura y Deporte: Secretaría General Técnica: Subdirección General de Documentación y Publicaciones. Disponible en: https://bit.ly/3hU60GZ.

García-Restrepo, C. (2014). Más allá de la hermenéutica y la mayéutica: el pensamiento creativo. Medellín: Universidad de Antioquia.

González Jiménez, F. E. (Dir.) (2010). Selección formación y práctica de los docentes investigadores. La carrera docente. Madrid: Universitas.

Gutiérrez-Braojos, C. y Salmerón, H. (2012). Estrategias de comprensión lectora: Enseñanza y evaluación en educación primaria. Profesorado. Revista de Currículum y Formación del Profesorado, 16, 183-202.

Guzmán, B., Véliz, M., \& Reyes, F. (2017). Memoria operativa, comprensión lectora y rendimiento escolar. Literatura y lingüística, (35), 377-402

Halpern, D. F. (1984). Thought and Knowledge: An Introduction to Critical Thinking. Hillsdale. NJ: Erlbaum Associates.

Hines, S. J. (2009). The Effectiveness of a Color-Coded, Onset-Rime Decoding Intervention with First- Grade Students at Serious Risk for Reading Disabilities. Learning disabilities research and practice. 24 (1), 21-32. Jacoboni M, Woods R. P, Brass M, Bekkering H, Mazziotta J. C \& Rizzolatti G. (1999). Cortical mechanisms of human imitation. Science; 286: 2526-8.

Jolliffe, D. \& Farrington, D. P. (2006). Development and validation of the Basic Empathy Scale. Journal of Adolescence, 29, 589-611. Doi: 10.1016/j.adolescence.2005.08.010

Lee, L., Harrison, L. M., \& Mec-helli, A. (2003). A report of the functional connectivity workshop, Dusseldorf 2002. Neuroimage, 19(2), 457-465

Lenoir y Morales-Gómez, M.A (2011). El enfoque por competencias y profesionalización de la enseñanza: una clarificación conceptual. Revista Iberoamericana sobre Calidad, Eficacia y Cambio en Educación, 1(9), 46-64. Ley Orgánica 8/2013, de 9 de diciembre, para la Mejora de la Calidad Educativa. BOE, ํㅜ 295, de 10 de diciembre de 2013. Real Decreto 126/2014, de 28 de febrero, por el que se establece el currículo básico de la Educación primaria. BOE, no 52, de 1 de marzo de 2014.

Ley Orgánica 2/2006, de 3 de mayo, de Educación, en la redacción dada por la Ley Orgánica 8/2013, de 9 de diciembre, para la Mejora de la Calidad Educativa, dedica el Capítulo II del Título I a la regulación de la educación primaria.

López, V. (2015). Importancia de la valoración de la creatividad desde su base neuropsicológica. En P. Martín Lobo. Procesos e instrumentos de evaluación neuropsicológica educativa (pp. 140-162). Madrid: Ministerio de Educación, Cultura y Deporte: Secretaría General Técnica: Subdirección General de Documentación y Publicaciones. Disponible en: https://bit.ly/3ggbjAf.

Martín-Lobo, P. (2015). La intervención desde la base neuropsicológica y metodologías que favorecen el rendimiento escolar. En P. Martín-Lobo (Coord.), Procesos y programas de neuropsicología educativa (pp. 1432). Madrid: CNIIE.

Martínez, I., (2015). Procesos y Programas neuropsicológicos para la lectura. En P. Martín Lobo. Procesos y programas de neuropsicología educativa (pp. 114-122). Madrid: Ministerio de Educación, Cultura y Deporte: Secretaría General Técnica: Subdirección General de Documentación y Publicaciones. Disponible en: https://bit.ly/39JettC

McClelland, D. (1973). Testing for competence rather than for intelligence. American Psychologist, 28, 1-14. Oliva, A., Antolín, L., Pertegal, M., Ríos, M., Parra, A., Hernando, A. y Reina, M. (2011). Instrumentos para la evaluación de la salud mental y el desarrollo positivo adolescente y los activos que lo promueven. Sevilla: Consejería de Salud.

Omerger, G. (2002). Enciclopedia de Recursos Humanos. Madrid: Artegraf.

Pradas, S. y De la Peña, C. (2015). Programas para superar las dificultades del aprendizaje. En P. Martín Lobo (Coord.), Procesos y programas de neuropsicología educativa (pp. 187-199). Madrid: CNIIE.

Retuerto, A. (2004). Diferencias en empatía en función de las variables género y edad. Apuntes de psicología, 22(3), 323-339.

Resolución de 20 de mayo de 2016, de la Dirección General de Política Educativa Escolar de la Junta de Castilla y León

Revuelta, L. R., Rodríguez, A., Ormaza, U. R., y Ramos, E. (2016). Autoconcepto multidimensional: medida y relaciones con el rendimiento académico. Revista Internacional de Evaluación y Medición de la Calidad Educativa, 2(1), 12-25

Rico Martín, A.M y Níkleva, D. (2016). Análisis de la competencia lingüístico-discursiva escrita de los alumnos de nuevo ingreso del Grado de Maestro en Educación Primaria. Revista signos, 49(90), 48-70. DOI: 10.4067/S071809342016000100003.

Rodríguez-Fernández, A., Ramos-Díaz, E., de Lahidalga, I. R. M. y Rey-Baltar, A. Z. (2018). Implicación escolar de estudiantes de secundaria: La influencia de la resiliencia, el autoconcepto y el apoyo social percibido.

Educación XX1, 21(1), 87-108 
Sánchez, F., y Fiol, M. L. (2016). Creatividad matemática: Momentos de insight en estudiantes de 4ํ de ESO. Journal of Research in Mathematics Education, 5(1), 28-55.

Samamé, M. (2014). Comprensión lectora y rendimiento académico en los estudiantes del primer ciclo de la Escuela Académico Profesional de Educación de la Universidad Alas Peruanas. Convergencia Científica, 1(1), 73-76

Simonton, D. K. (2000). Creativity: cognitive, personal, developmental and social aspects. American Psychologist, $55,151-158$.

Shavelson, R.J., Hubner, J.J., \& Stanton, G.C. (1976). Self-concept: Validation of construct interpretations. Review of Educational Research, 46(3), 407-441. Doi:10.2307/1170010.

Solé, I. (2001). Comprensión lectora. El uso de la lengua como procedimiento. Barcelona: Grao.

Tettamanti M., Giovanni, B., Saccuman, Ma C., Gallese, V., Danna, M., Scifo, P... Perani, D. (2005). Listening to action-related sentences activates fronto-parietal motor circuits. Journal of Cognitive Neuroscience, 17, $273-81$. Wallas, G. (1926). The Art of Thought. New York: Harcourt Brace. 